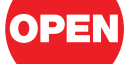

SUBJECT AREAS:

IMMUNOLOGY

NANOBIOTECHNOLOGY

SYNTHETIC BIOLOGY

BIOTECHNOLOGY

Received

23 August 2011

Accepted

3 October 2011

Published

19 October 2011

Correspondence and requests for materials should be addressed to R.P. (richard.pleass@ liv.ac.uk)

* These authors contributed equally to this work.

\title{
Polymeric human Fc-fusion proteins with modified effector functions
}

\author{
David N. A. Mekhaiel ${ }^{*}$, Daniel M. Czajkowsky ${ }^{2,3 *}$, Jan Terje Andersen ${ }^{4,5 *}$, Jianguo Shi' \\ Marwa El-Faham ${ }^{6,7}$, Michael Doenhoff 6 , Richard S. Mclntosh ${ }^{6}$, Inger Sandlie ${ }^{4}$, Jianfeng $\mathrm{He}^{8}$, Jun $\mathrm{Hu}^{2}$, \\ Zhifeng Shao ${ }^{3}$ \& Richard J. Pleass ${ }^{1}$
}

'Liverpool School of Tropical Medicine, Pembroke Place, Liverpool, L3 5QA, UK, ${ }^{2}$ Shanghai Institute of Applied Physics, Chinese Academy of Sciences, Shanghai 200240, China, ${ }^{3}$ Key Laboratory of Systems Biomedicine (Ministry of Education), Shanghai Jiao Tong University, Shanghai 200240, China, ${ }^{4}$ Centre for Immune Regulation and Department of Molecular Biosciences and Centre for Immune Regulation, University of Oslo, P.O. Box 1041, N-0371 Oslo, Norway, ${ }^{5}$ Department of Immunology, Oslo University Hospital Rikshospitalet and University of Oslo, PO Box 4956, N-0424 Oslo, Norway, ${ }^{6}$ School of Biology, University of Nottingham, Nottingham, NG7 2RD, ${ }^{7}$ University of Alexandria, Alexandria, Egypt, ${ }^{8}$ Department of Biomedical Engineering, Shanghai Jiao Tong University, Shanghai, China.

The success of Fc-fusion bio-therapeutics has spurred the development of other Fc-fusion products for treating and/or vaccinating against a range of diseases. We describe a method to modulate their function by converting them into well-defined stable polymers. This strategy resulted in cylindrical hexameric structures revealed by tapping mode atomic force microscopy (AFM). Polymeric Fc-fusions were significantly less immunogenic than their dimeric or monomeric counterparts, a result partly owing to their reduced ability to interact with critical Fc-receptors. However, in the absence of the fusion partner, polymeric IgG1-Fc molecules were capable of binding selectively to Fc $\gamma$ Rs, with significantly increased affinity owing to their increased valency, suggesting that these reagents may prove of immediate utility in the development of well-defined replacements for intravenous immunoglobulin (IVIG) therapy. Overall, these findings establish an effective IgG Fc-fusion based polymeric platform with which the therapeutic and vaccination applications of $\mathrm{Fc}$-fusion immune-complexes can now be explored.

rotein therapeutics has recently emerged as a significant class of highly effective pharmaceuticals, and within this group, Fc-based fusion proteins, in which the Fc domain of an antibody (Ab) of the IgG isotype is joined to a different protein, are considered among those with the greatest promise ${ }^{1,2}$. Already with demonstrable successes $^{1,2}$, their effectiveness is commonly believed to be owing to interactions with specific effector proteins, such as the salvage receptor FcRn that increases serum half-life and prolongs therapeutic activity ${ }^{3,4}$. Similarly, the $\mathrm{Fc}$ region of Abs is increasingly being explored as an Ag delivery vehicle in adjuvants for the induction of protective immunity or tolerance owing to their ability to activate specific Fc $\gamma$ Rs that trigger activating and/or inhibitory signalling pathways that set thresholds for well-balanced immune responses ${ }^{5}$. However, existing approaches that use the Fc to deliver Ag to immune cells have one major drawback that stems from their monomeric structure, namely an inability to cross-link multiple Fc $\gamma$ Rs with the high affinity required for enhanced cell signalling.

In particular, monomeric Fc-fusions bind with negligible avidity to key low-affinity Fc $\gamma$ Rs, including Fc $\gamma$ RIIA, Fc $\gamma$ RIIB, and Fc $\gamma$ RIII that are crucial to initiating protective immune responses ${ }^{6,7}$. Most Fc $\gamma$ Rs (with the exeption of Fc $\gamma$ RI) can only interact with high avidity to Abs that are presented to the immune system as immune complexes (ICs). Several studies have shown that ICs are potent activators of dendritic cells (DCs) and can prime stronger immune responses than $\mathrm{Ag}$ or $\mathrm{Ab}$ alone $\mathrm{e}^{8-12}$. Importantly, $\mathrm{Fc} \gamma \mathrm{R}$-dependent IC internalization not only results in MHC-class-II-restricted Ag presentation, but also in cross-presentation on MHC class I molecules, thereby priming both $\mathrm{CD}^{+}$and $\mathrm{CD}^{+} \mathrm{T}$-cell responses ${ }^{8}$. The magnitude of these responses are controlled and enhanced in part by $\mathrm{FCRn}^{13}$, but also by the inhibitory Fc $\gamma$ RIIB, as DCs derived from Fc $\gamma$ RIIB-knock out mice generate stronger and longer-lasting immune-responses in vitro and in vivo ${ }^{14,15}$. More importantly, Fc $\gamma$ RIIBdeficient DCs, or DCs incubated with a mAb that blocks IC binding to Fc $\gamma$ RIIB, show a spontaneous maturation $^{16,17}$, suggesting that the inhibitory Fc $\gamma$ RIIB not only regulates the magnitude of cell activation, but also actively prevents spontaneous DC maturation under non-inflammatory steady-state conditions ${ }^{16,17}$. 

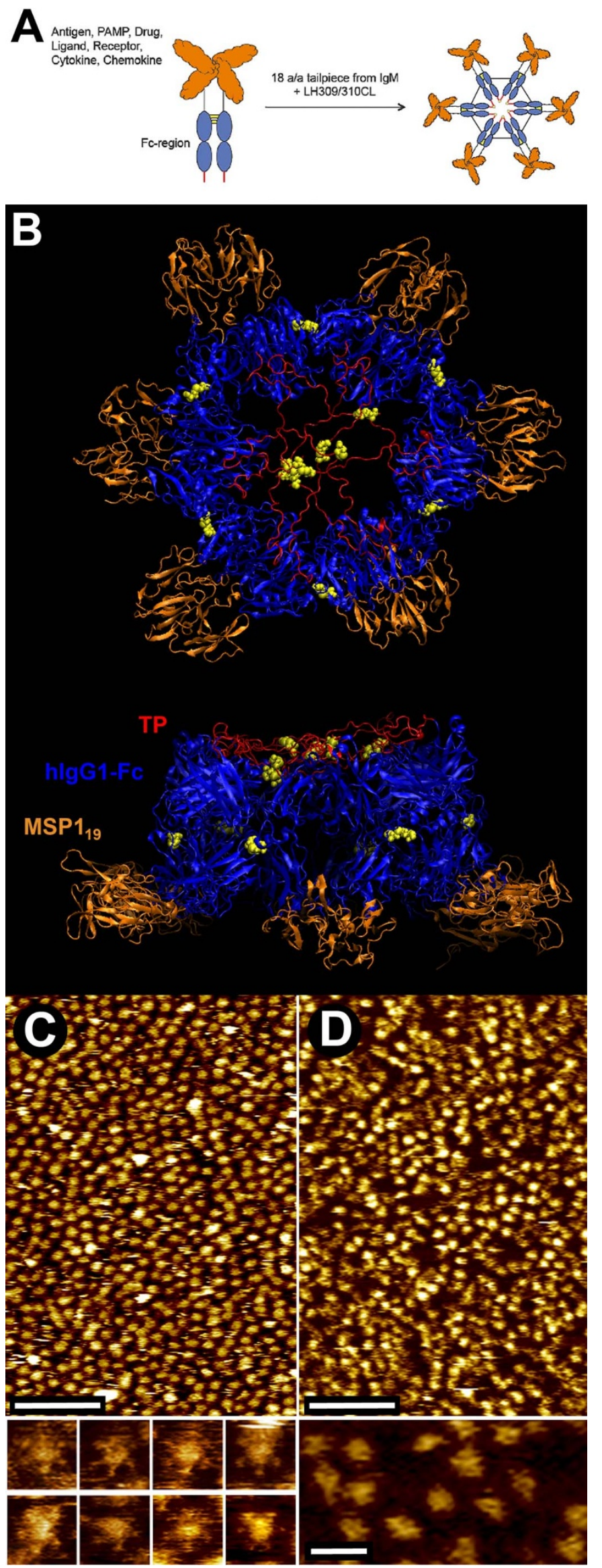

Figure $1 \mid$ Hexameric Fc-fusions and their structural characterization (a) Schematic diagram illustrating the novel approach by which Fc-fusion
Thus, it has been a long sought goal of biosynthetic immunology to develop a strategy to efficiently couple monomeric IgG Fc-fusion proteins into polymeric ICs so as to take advantage of the existing intrinsic host pathways optimized for native ICs. While native ICs can contain many Fc domains owing to the binding of many Abs to the same antigenic complex, this means of multimerization is not possible with $\mathrm{Fc}$-fusions owing to the covalent linkage of the Ag to the Fc domain. Here, instead, we use the knowledge of how IgM polymerizes via their Fc domains to develop a novel approach for generating recombinant polymeric Fc-fusion proteins (Figure 1). We show that this strategy is effective with a human IgG1-Fc subclass and results in a significantly increased affinity (improved binding), likely resulting from the increased avidity, for both Fc $\gamma$ Rs and FcRn. Yet we also identify a surprising dependence of this interaction on the fusion partner, which provides useful guidance for future developments. Finally we characterize the immunogenicity and immunotolerance of these first-generation constructs in mouse models of malaria and schistosomiasis, and identify properties that require further development to more fully meet their vaccination potential. Nonetheless, we identify a number of constructs that for certain clinical applications, in particular IVIG therapy, would have significant advantages over existing strategies and thus could be of immediate benefit. Thus in total, this work provides a generally applicable strategy to construct polymeric IgG Fc-fusions with which the therapeutic and vaccination potential of recombinant ICs can now be investigated.

\section{Results}

Production of Fc-fusion proteins. To first establish a general framework with which polymeric Fc-fusion proteins can be produced to high yield, we first developed procedures for the production of monomeric Fc-fusions. In particular, we fused the Fc of human IgG1 (hIgG1) or mouse IgG2a (mIgG2a) to a wide variety of proteins and glycoproteins (See supplementary Table S1 online), including the 19 and $42 \mathrm{kDa}$ subunit Ags from Plasmodium falciparum $(P f)$ or Plasmodium yoelii $(P y)$ merozoite surface protein

proteins can be polymerized. Attaching the C-terminal IgM tailpiece and mutating Leu 309 to Cys to enable formation of inter-monomeric disulfide bridges produces well-defined hexameric Fc-fusion complexes. (b) Molecular model of the hexameric PfMSP $1_{19}$-hIgG1Fc-TP-LH309/310CL. After first energy minimizing the monomer, the hexameric model was constructed by rotating each monomer by multiples of $\sim 60^{\circ}$, keeping the C309 residues (here C207) within a common plane, followed by linking together neighboring monomers by these cysteines and minimizing the energy and equilibration. Shown are top and side views of the hexamer with the C309 and tailpiece cysteine (here, C366) residues as yellow vdW spheres. The $\mathrm{C} 309$ residues in neighboring monomers are covalently linked to each other in this model. The tailpiece cysteines in randomly selected monomers were also joined together. The tailpieces are shown in red, the Fc of hIgG1 in blue, and PfMSP $1_{19}$ in orange. (c) AFM images of PfMSP $1_{19}$-hIgM-Fc reveal star-shaped, six-fold symmetric complexes. At larger scan sizes (top panel), the sample shows a uniform population of circular complexes. At smaller scan sizes (lower panel), these complexes are shown to consist of a central protrusion $\sim 20 \mathrm{~nm}$ in diameter, around which are arranged predominantly six, symmetrically disposed smaller radial projections. The scale bar in the top panel is $200 \mathrm{~nm}$ and each of the images in the lower panel is $75 \times 75 \mathrm{~nm}^{2}$. (d) AFM images of hIgG1-FcLH309/310CL-TP show structures consistent with hexameric complexes. The larger scan size image (top panel) shows a highly uniform population of circularly shaped complexes. The smaller scan sized images show these complexes to consist of a circular structure that is similar in diameter and height to the central protrusion of the PfMSP $1_{19}$-hIgM-Fc hexamers, as expected for hexameric Fc-fusion complexes without the fused partners. The scale bars in the top and lower panels are $200 \mathrm{~nm}$ and $50 \mathrm{~nm}$, respectively. 
1 (MSP1), and expressed these Fc-fusions in CHO-K1 or HEK cell lines widely used for good GMP-production of therapeutic Abs by the pharmaceutical sector. These cells expressed good yields of the Fc-fusions that were exported from the cell, allowing for easy and cost-effective purification on protein-G sepharose columns to which the proteins were bound. These Fc-fusion proteins all ran as monomers by size-exclusion chromatography (SEC) and Western blotting (See supplementary Fig.1 online).

Difficulties in manufacturing native ICs have limited studies of their therapeutic and vaccination potential applications. In particular, natural ICs are both ill-defined (ratio of the Ab: Ag for use) and unstable, both of which are significant obstacles for any systematic investigation. We sought to overcome these shortcomings by engineering the monomeric Fc-fusions into recombinant ICs as depicted in Fig. 1A. It has been shown that the 18 amino acids located at the carboxyl-terminus of human IgM (hIgM) are involved in polymer assembly, as this single modification has been shown to generate large hIgG polymers with increased effector function ${ }^{18}$. We added the 18 amino acid tailpiece (-tp) from hIgM together with a 5 aminoacid linker to the C-terminus of the human and mouse IgG Fcfusions. However, in the context of Fc-fusions studied here, this single modification did not induce polymerization of either the hIgG1-Fc or mIgG2a-Fc backbones, which were expressed entirely as monomers (See supplementary Table 1).

Previously, we demonstrated the importance of a cysteine residue at position 309 for the formation of polymeric $\mathrm{Abs}^{19,20}$. The homologous Cys414 in hIgM is known to form inter-monomeric disulfide bridges that are critical for its polymerization into large molecular weight complexes, which, in the absence of J chain (as is the case with the $\mathrm{CHO}-\mathrm{K} 1$ cells), are hexamers. We therefore used site-directed mutagenesis to introduce this mutation into the equivalent position in the -tp containing monomeric Fc-fusion proteins. We also mutated the neighboring His310 to Leu, since a histidine at this position might have inhibited polymeric assembly by interfering with disulphide bridge formation. This final modification allowed the hIgG1 based Fc-fusions to polymerize into molecules of $\sim 540 \mathrm{kDa}$ when analyzed by SEC or western blotting (See supplementary Fig. 2 online), and by comparison with the hIgM-Fc fusions which acted as controls for polymerization (See supplementary Fig. 3 online). A molecule of this size would represent a hexameric conformation of the Fc-fusion protein as shown in Fig. 1. We also observed a protein of $\sim 160 \mathrm{kDa}$ that likely represents a dimeric conformation of the hIgG1-based construct (See supplementary Fig. 2 online), since it too was detected by immunoblotting with Fc-specific reagents (See supplementary Fig. 2 online). When the polymeric Fc-fusion constructs are expressed in the absence of Ag, we observed proteins of $\sim 312$ and $\sim 100 \mathrm{kDa}$ consistent with these also being expressed as hexameric and dimeric entities (See supplementary Fig. 2 online). Interestingly, these same modifications in the mIgG2a backbone failed to allow us to purify anything larger than a dimer by protein- $G$ affinity chromatography. There was no obvious degradation of PfMSP1 19 -hIgG1-Fc-LH309/310CL-TP or the $P y \mathrm{MSP} 1_{19}$-hIgM-Fc after long-term storage $(>4$ months) at either $4^{\circ} \mathrm{C}$ or $-20^{\circ} \mathrm{C}$, which was confirmed by SEC and SDSPAGE gel analysis.

Structure of hexameric Fc-fusion proteins. To determine if a hexameric architecture could be accomodated by the human IgG1Fc backbone when fused to Ag, we modelled the PfMSP1 19 -hIgG1Fc-LH309/310CL-TP hexamer following principles similar to those applied with the recently determined structure of polymeric hIgM ${ }^{21}$. From this model, it is apparent that a molecule as large as the $19 \mathrm{kDa}$ PfMSP $_{19}$ Ag could easily be accomodated within this polymeric platform (Figure 1b). Furthermore, analysis of this model confirms that the protein $\mathrm{G}, \mathrm{FcRn}$ and $\mathrm{Fc} \gamma \mathrm{R}$ binding sites would not be occluded with this polymerization (See supplementary Fig. 4 online). These hexamers, however, did exhibit some flexibility in the lower hinge region, at the junction of $\mathrm{Ag}$ attachment, as might be expected for this region, which could influence the binding of receptors.

To directly characterize the size and morphology of the hexameric Fc-fusions, we imaged PfMSP1 19 -hIgM-Fc and hIgG1-Fc-LH309/ 310CL-TP constructs with tapping mode AFM under solution. Hexameric PfMSP1 19 -hIgG1-Fc-LH309/310CL-TP complexes failed to bind to mica substrate with sufficient strength to withstand the lateral forces imparted by the scanning tip, even in the tapping mode. As shown in Fig. 1C, both polymeric fusion complexes form a highly uniform population of well-defined structures. With the PfMSP1 $1_{19^{-}}$ hIgM-Fc polymers, the AFM images reveal a central protusion $(20 \pm$ $3 \mathrm{~nm}(\mathrm{n}=46)$ in diameter, $5.8 \pm 0.5 \mathrm{~nm}(\mathrm{n}=52)$ in height $)$ surrounded by predominantly six, hexagonally arranged, smaller radial projections, consistent with the expected hexameric architecture of these proteins and previous cryo-AFM images of native $\operatorname{IgM}^{21}$. With the hIgG1-Fc-LH309/310CL-TP construct (Figure 1d), the AFM images show the complexes as cylindrical structures $(18 \pm 2 \mathrm{~nm}(\mathrm{n}=51)$ in diameter, $5.7 \pm 0.4 \mathrm{~nm}(\mathrm{n}=54)$ in height $)$, similar to the dimensions of only the central protrusion in the hIgM-Fc hexamers. These images are also consistent with the dimensions predicted from the aforementioned modelling analysis (Figure 1B). Thus, overall, these direct images of the constructs verify that these IgG Fc-fusions are well-folded and readily self-assembled into predominantly hexameric polymers.

Binding to FcyRs. Since native ICs are functionally distinguished from monomeric Fc-fusions by a significantly higher affinity for many Fc $\gamma$ Rs, we tested whether these recombinant ICs would show a likewise stronger affinity for these receptors relative to their monomeric counterparts. The monomeric Fc-fusions produced here bound to both human (Figure 2a) and mouse Fc $\gamma$ Rs (Figure 2b) with the low affinity expected of monomers. Unexpectedly however, the nature of the molecule fused to the Fc markedly affected the ability of the monomeric Fc-fusions to interact with some of the FcRs. In particular, while all of the fusions bound to both human and mouse high affinity Fc $\gamma$ RI with only minor differences in affinity, the hIgG1-Fc fusion to the large $42 \mathrm{kDa} \mathrm{Ag}\left(P y \mathrm{MSP}_{42}\right.$-hIgG1-Fc) bound to hFc $\gamma$ RIIA $^{\mathrm{H} 131}$ and hFc $\gamma$ RIIIA/B, while the smaller $19 \mathrm{kDa}$ PfMSP1 $1_{19}$-hIgG1-Fc fusion bound poorly to both receptors. The reasons for these differences are not clear, but it does indicate a property that must be taken into consideration in the future design of monomeric Fc-fusions.

With the polymeric Fc-fusions, in stark contrast to either the monomeric PfMSP1 19 -hIgG1-Fc (Figure 2) or the dimeric PfMSP1 19 -hIgG1-Fc-LH309/310CL-TP (which bound hFc $\gamma$ RI, hFc $\gamma$ RIIA $^{\text {R131 }}$, hFc $\gamma$ RIIA $^{\text {H131 }}$, hFc $\gamma$ RIIB, but not Fc $\gamma$ RIIIA/B), the hexameric PfMSP1 19 -hIgG1-Fc-LH309/310CL-TP bound only weakly to hFc $\gamma \mathrm{RI}$ and was unable to bind to any of the other human or mouse Fc $\gamma$ Rs (Figure 3). However, this lack of binding to Fc $\gamma$ Rs was due to the presence of the PfMSP1 $1_{19}$, since hexameric hIgG1-FcLH309/310CL-TP bound very well to all of the Fc $\gamma$ R investigated here (Figure 3). In fact, the hIgG1-Fc-LH309/310CL-TP hexamers bound with a log-order higher affinity to hFc $\gamma$ RI and hFc $\gamma$ RIIA ${ }^{\mathrm{R} 131}$, as expected from its increased valency (Figure 3 and supplementary Fig. 5 online). Also in contrast to hIgG1-Fc monomers, there was marked binding of the hexameric hIgG1-Fc-LH309/310CL-TP to both inhibitory human and mouse Fc $\gamma$ RIIB (Figure 3). Overall then, these results thus indicate that this means of polymerization produces complexes that can indeed exhibit the high affinity for Fc $\gamma$ Rs characteristic of native ICs.

Binding to the neonatal FcRn. We next investigated the ability of the monomeric Fc-fusions to interact with the neonatal FcRn, which is responsible for maintaining the long half-life of Abs in the circulation. The monomeric hIgG1 Fc-fusions interacted almost 
A
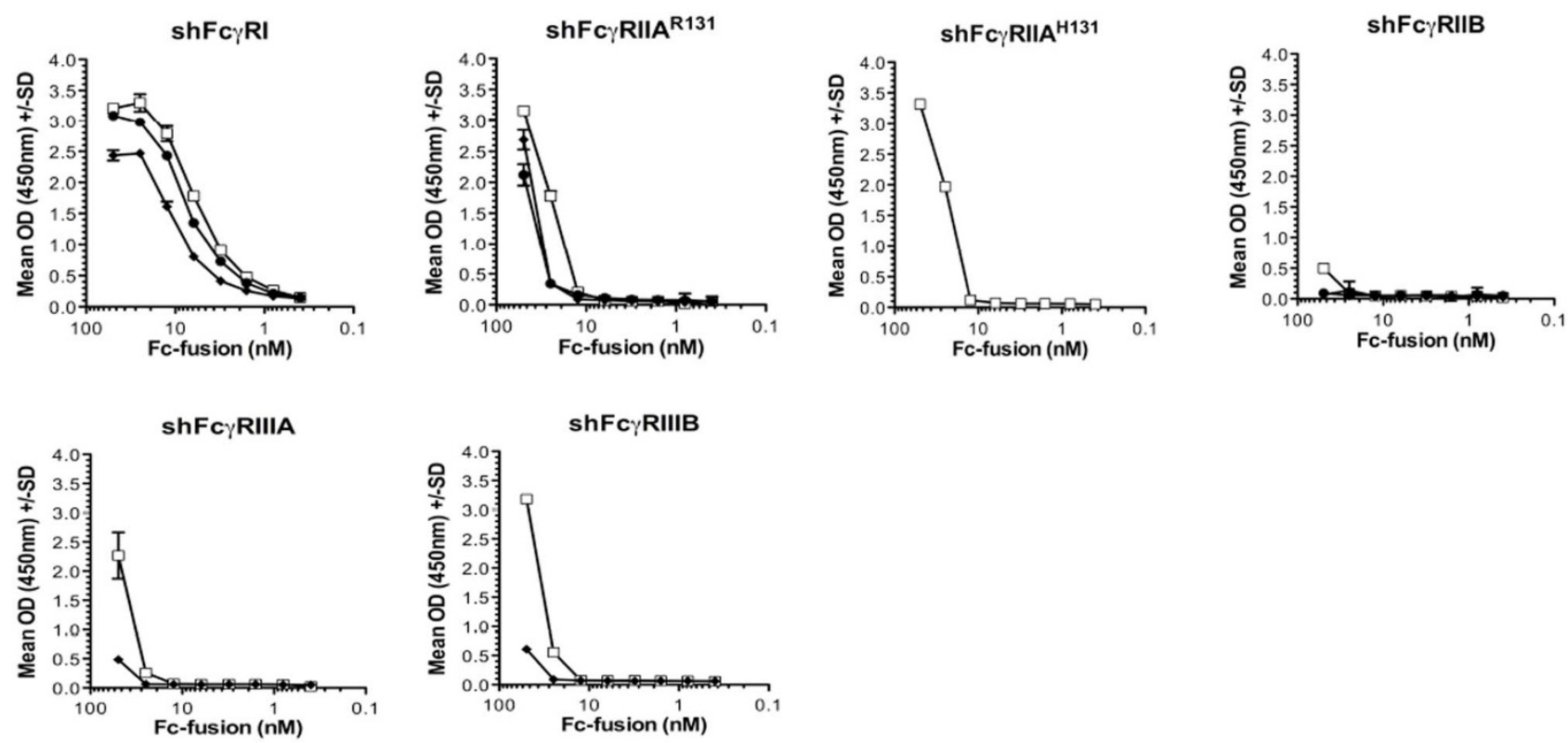

B
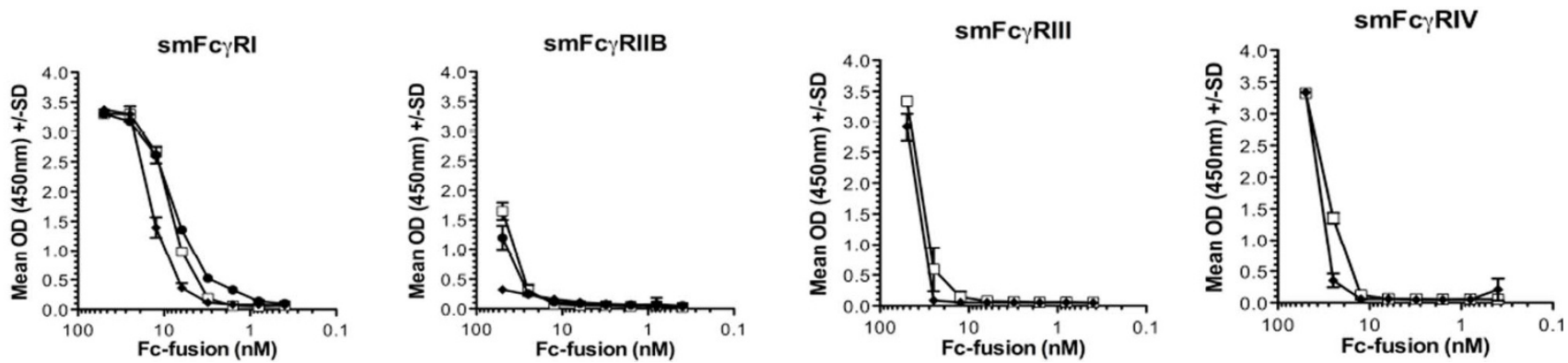

Figure $2 \mid$ Binding of monomeric Fc-fusions to human and mouse Fc $\gamma$ Rs. Titrated amounts (50-0.3 nM) of Fc-fusion proteins were coated onto ELISA wells as described in methods. Binding of GST-fused (a) human and (b) mouse Fc $\gamma$ Rs as indicated were visualized using an HRP-conjugated anti-GST Ab. $(\bullet)=P f \mathrm{MSP}_{19}-\mathrm{hIgG1}-\mathrm{Fc},(\square)=P y \mathrm{MSP}_{42}$-hIgG1-Fc, and $(\bullet)=P f \mathrm{MSP}_{19}$-mIgG2a-Fc. The values represent the average of triplicate determinations.

equally well, and in a pH dependent manner with human FcRn in SPR analysis (Figure $4 \mathrm{a}$ ). The murine PfMSP1 19 -mIgG2a-Fc bound only weakly to the human FcRn, in contrast with the human based Fc-fusions that bound with higher affinity to the mouse FcRn (Figure $4 \mathrm{~b}$ ), and in all cases binding only occurred at pH6.0, as expected from previous observations ${ }^{22}$.

With the polymers, similar to the observations with Fc $\gamma$ Rs, hexameric PfMSP1 19 -hIgG1-Fc-LH309/310CL-TP failed to bind to either hFcRn or $\mathrm{mFcRn}$, in contrast to the dimer PfMSP1 $1_{19}$-hIgG1Fc-LH309/310CL-TP which bound weakly to hFcRn and more strongly to $\mathrm{mFcRn}$ as determined by SPR analysis (Figure 5). In the absence of the MSP $1_{19} \mathrm{Ag}$, the hexameric hIgG1-Fc was capable of binding $\mathrm{mFcRn}$ but not $\mathrm{hFcRn}$ suggesting that the presence of the Ag also markedly affected binding to FcRn across species (Figure 5). The half-life of PfMSP1 $_{19}$-hIgG1-Fc-LH309/310CL-TP was significantly shorter $(\sim 18 \mathrm{~h})$ than for IVIG (predominantly monomeric $\mathrm{IgG}$ ) when injected i.p. into mice, a result that was predicted from the lack of binding of PfMSP1 19 -hIgG1-Fc-LH309/310CL-TP to mFcRn (See supplementary Fig. 6 online).

Binding to complement. Because natural ICs may activate complement, we also investigated the ability of $P f M S P 1_{19}$-hIgG1Fc-LH309/310CL-TP hexamers and dimers to bind $\mathrm{Clq}$ and activate the classical complement pathway (See supplementary Fig. 7). Hexamers bound C1q poorly compared to dimers, which in turn bound less well than intact monomeric IgG, a gradation reflected in comensurate decreases in detection of the C5-9 terminal complex. The absence of Ag did not markedly improve binding of either dimer or hexamer to $\mathrm{Clq}$ (data not shown), and both C1q binding and C5-9 deposition were negligible for the control IgA Ab (See supplementary Fig. 7). Taken together with the lack of binding to Fc $\gamma$ Rs, the reduced complement activation seen in these in vitro experiments is supported by the complete lack of an Arthus reaction, a type III hypersensitivity reaction initiated by IC formation at the site of inoculation, even when PfMSP1 ${ }_{19}$-hIgG1-Fc-LH309/ 310CL-TP was administered with alum (see Immunization with Fcfusion proteins).

Enhanced binding of polymeric hIgG1 Fc-fusions to $B$ cells. As a first step to evaluate the interaction of these polymeric Fc-fusions with immune cells involved in Ag presentation, we investigated their ability to bind to CD $19^{+}$B cells by FACS analysis. Since these cells only express Fc $\gamma \mathrm{RIIB}^{5-7}$, we expected that hexameric PfMSP1 ${ }_{19^{-}}$ hIgG1-Fc-LH309/310CL-TP would bind less strongly than the corresponding dimers, in accordance with the aforementioned ELISA and SPR results. However, as shown in (Figure 6), the hexamers were found to bind to a greater degree to these $\mathrm{CD} 19^{+} \mathrm{B}$ cells than the dimers. Thus, the results indicate that there is a receptor on these cells (not Fc $\gamma$ RIIB) that interacts with polymeric PfMSP1 $1_{19^{-}}$ hIgG1-Fc-LH309/310CL-TP constructs, and further suggests that the greater affinity for this unidentified receptor for the hexamers, is owing to its greater valency. 
A

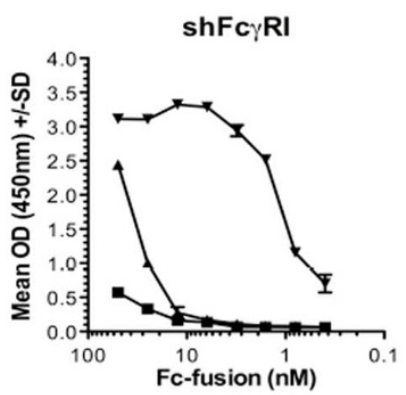

B

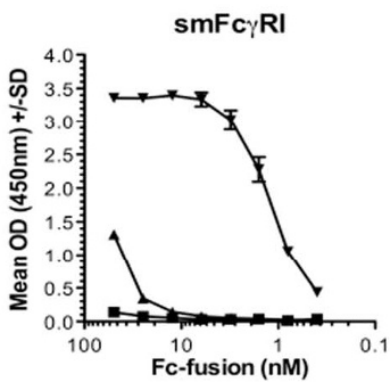

ShFc $\gamma$ RIIA R131

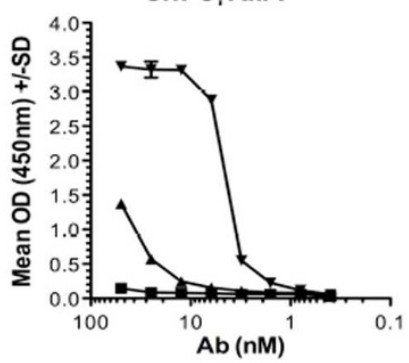

smFc $\gamma$ RIIB

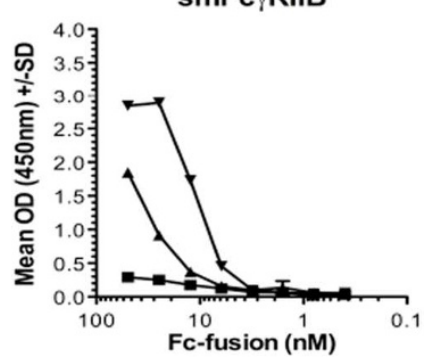

shFc $\gamma$ RIIA $^{\mathrm{H} 131}$

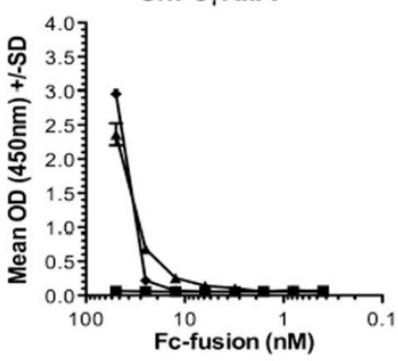

smFc $\gamma$ RIII

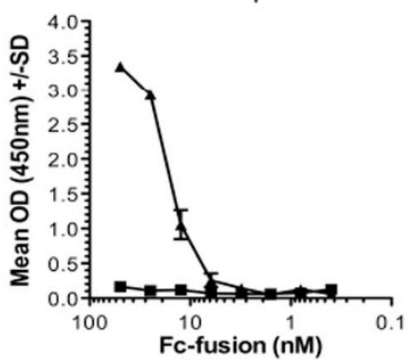

shFcyRIIB

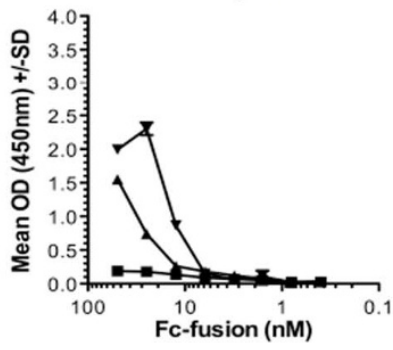

smFc $\gamma$ RIV

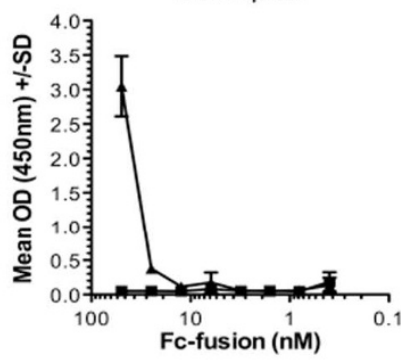

Figure 3 Binding of polymeric Fc-fusions to human and mouse Fc $\gamma$ Rs. Titrated amounts (50-0.3 nM) of Fc-fusion proteins were coated onto ELISA wells. Binding of GST-fused (a) human and (b) mouse Fc $\gamma$ Rs receptors as indicated were visualized using an HRP-conjugated anti-GST Ab. $(\boldsymbol{\square})=$ hexamer PfMSP1 19 -hIgG1-Fc-LH309/310CL-TP, $(\mathbf{\Delta})=$ dimer PfMSP1 $19_{19}$-hIgG1-Fc-LH309/310CL-TP, and $(\boldsymbol{\nabla})=$ hIgG1-Fc-LH309/310CL-TP. The values represent the average of triplicate determinations.

Immunization with Fc-fusion proteins. Having determined that these first generation recombinant ICs exhibit similar properties as native ICs with respect to their interaction with known effector systems, we sought to evaluate their immunogenicity by testing these constructs for their efficacy in producing protective immunity in mouse models of malaria. The monomeric Fc-fusions induced antigen (Ag)-specific IgG1 responses when injected into mice (See supplementary Table 1 \& supplementary Fig. 8 online).
A

PFMSP1 $_{19}$-hlgG1-Fc

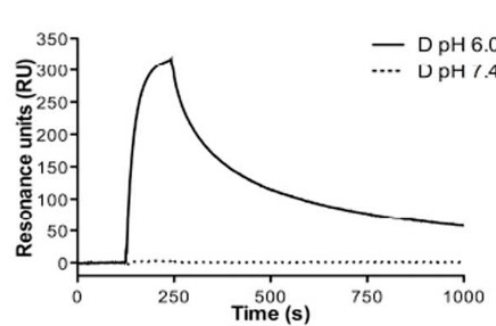

B PFMSP1 ${ }_{19}$-hlgG1-Fc

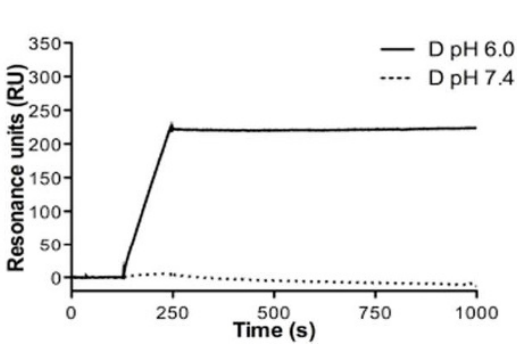

PfMSP1 $_{19}-$ mlgG2a-Fc

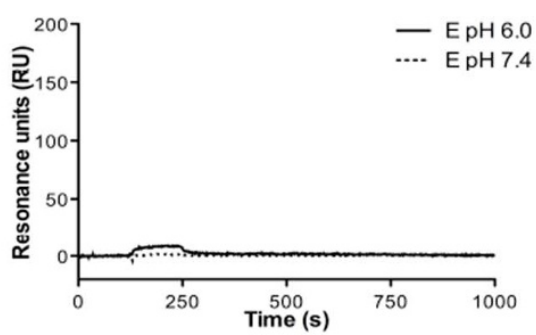

PfMSP1 $19-$ mlgG2a-Fc

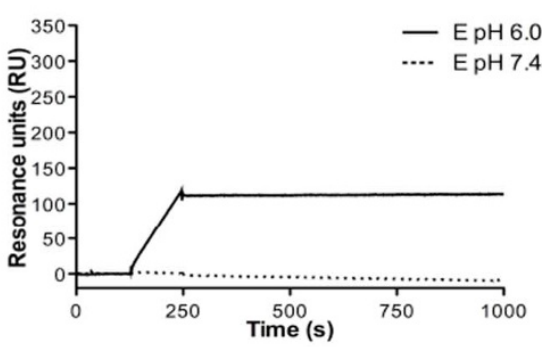

PyMSP1 $_{42}$-hlgG1-Fc

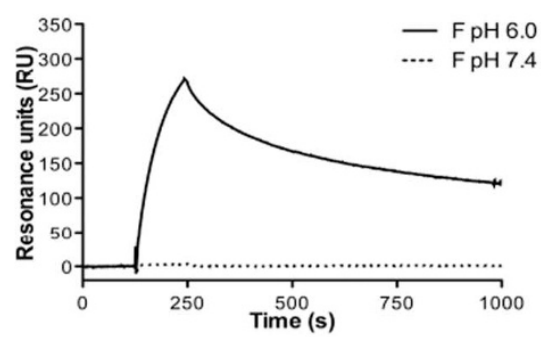

PyMSP1 $_{42}$-hlgG1-Fc

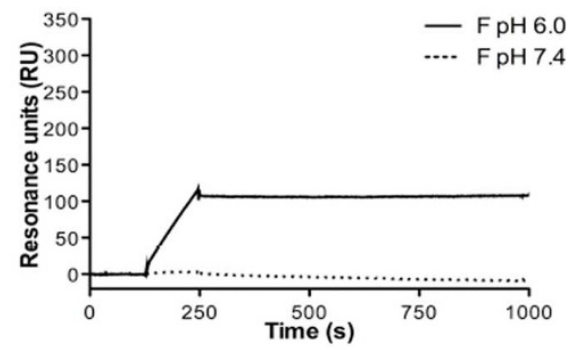

Figure 4 Binding of monomeric Fc-fusions to human and mouse FcRn. Representative sensograms showing binding of $100 \mathrm{nM}$ of each Fc-fusion variant injected over immobilized (a) hFcRn or (b) $\mathrm{mFcRn}$ at $\mathrm{pH} 6.0$ or $\mathrm{pH}$ 7.4. The experiments were performed at $25^{\circ} \mathrm{C}$ with a flow rate of $20 \mu \mathrm{l} / \mathrm{min}$. 
PfMSP1 $_{19}$-hlgG1-Fc-LH309/310CL-TP Dimer

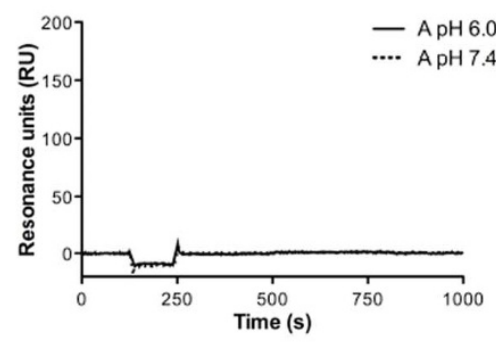

B PFMSP1 19 -hlgG1-Fc-LH309/310CL-TP Hexamer

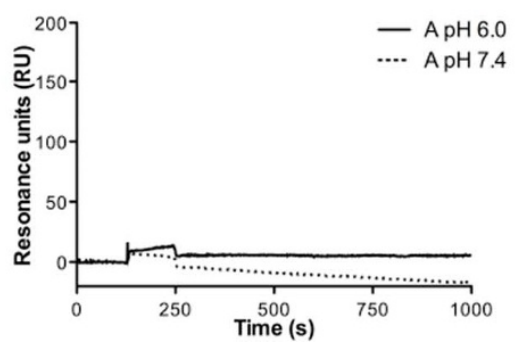

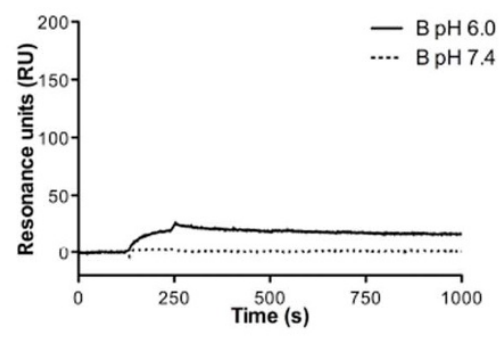

PfMSP1 ${ }_{19}$-hlgG1-Fc-LH309/310CL-TP Dimer

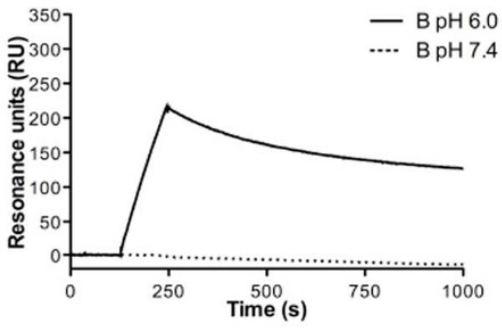

hlgG1-Fc-LH309/310CL-TP

Hexamer

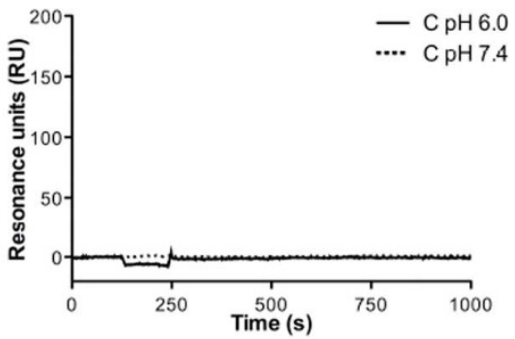

hlgG1-Fc-LH309/310CL-TP Hexamer

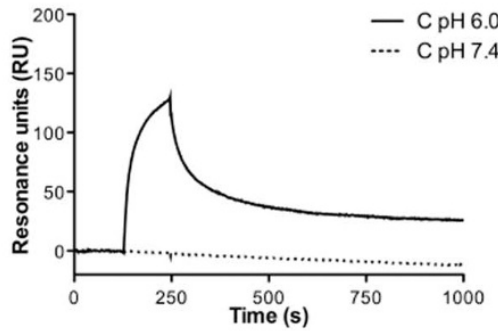

Figure 5 Binding of polymeric Fc-fusions to human and mouse FcRn. Representative sensograms showing binding of $100 \mathrm{nM}$ of each Fc-fusion variant injected over immobilized (a) hFcRn or (b) $\mathrm{mFcRn}$ at $\mathrm{pH} 6.0$ or $\mathrm{pH}$ 7.4. The experiments were performed at $25^{\circ} \mathrm{C}$ with a flow rate of $20 \mu \mathrm{l} / \mathrm{min}$.

Similar Ag-specific IgG1 responses were observed if PfMSP1 19 was fused to hIgG1-Fc or mIgG2a-Fc in immunizations of either wildtype or human Fc $\gamma$ RI (CD64) transgenic animals (See supplementary Fig. 8 online), a result supported by the ability of both variants to bind hFc $\gamma$ RI (Figure 2a). No drammatic improvement in Ab titres was obtained when monomeric Fc-fusions were co-administered with clinically relevant adjuvants e.g. alum (See supplementary Fig. 9 online), and this effect was maintained even after challenge with parasites. Prior immunization with the monomeric Fc-fusions also bolstered Ag-specific IgG responses by a log order during an infection with parasites (See supplementary Fig. 10 online). Despite high titres of $\mathrm{MSP} 1_{19}$-specific Ab $(1: 10,000)$ all animals inoculated with these Fc-fusions succumbed from malaria when challenged with parasites (See supplementary Table 1 online). Routes of Fc-fusion administration (s.c. vs i.p.) did not alter our basic findings, and although there were dose-dependent effects on overall titres with the PfMSP1 19 -mIgG2a-Fc monomer administered in alum (See supplementary Fig. 9 online), the course of the parasitemia did not significantly alter with different levels of Ab (See supplementary Table 1 online).

It should be noted that we obtained similar Ag-specific Ab titres to those seen with the PfMSP $1_{19}$-mIgG2a fusion using a monomeric mIgG2a-Fc fusion to the S. mansoni cercarial elastase (CE) Ag, a molecule that like $\mathrm{MSP}_{19}$ is non-immunogenic in its native form (See supplementary Table $1 \&$ supplementary Fig. 11 online $)^{23}$. In the presence of alum, immunization with a histidine-tagged CE (CEHis) construct generated consistently higher titres of CE-specific mIgG (all subclasses) compared to the CE-mIgG2a-Fc-fusion, a)

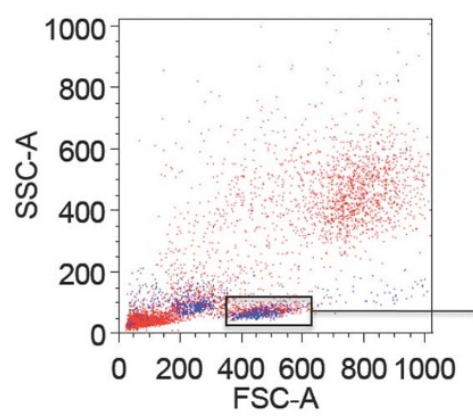

b)

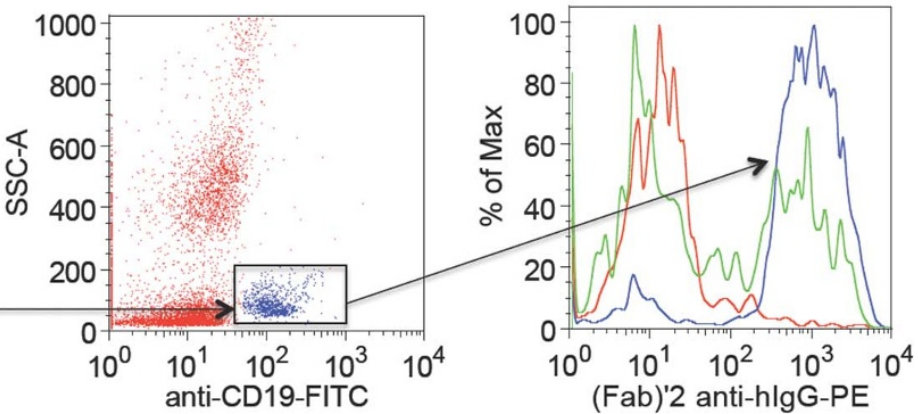

Figure 6 Binding of polymeric Fc-fusions to $\mathrm{CD} 19^{+}$human B-lymphocytes. (a) Characteristic FACs plot showing different populations of human leucocytes represented by their forward (FSC) and side (SSC) profiles. (b) Individual CD19 ${ }^{+}$B-lymphocytes stained with anti-human CD19-FITC (shown by blue dots) were gated. (c) Binding of $40 \mu \mathrm{g}$ of hexameric (blue trace) and dimeric (green trace) PfMSP $1_{19}$-hIgG1-Fc-LH309/310CL-TP to the gated human $\mathrm{CD}_{1} 9^{+} \mathrm{B}$ lymphocytes was detected using phycoerythrin (PE)-labeled goat $\left(\mathrm{Fab}_{2}{ }_{2}\right)$ anti-hIgG. No binding was detected with the phycoerythrin (PE)-labeled goat $\left(\mathrm{Fab}^{\prime}{ }_{2}\right)$ anti-hIgG in the absence of Fc-fusion proteins (red trace). Data are representative of three separate experiments. 
(a)

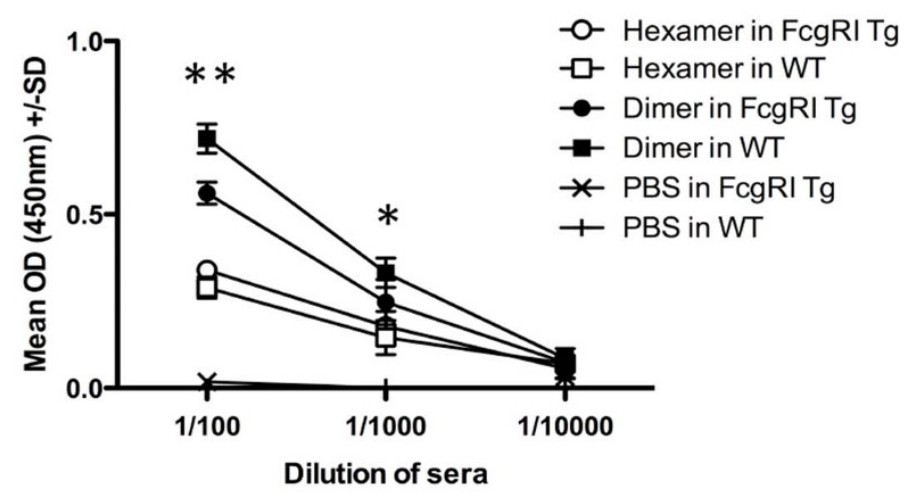

(b)

(c)
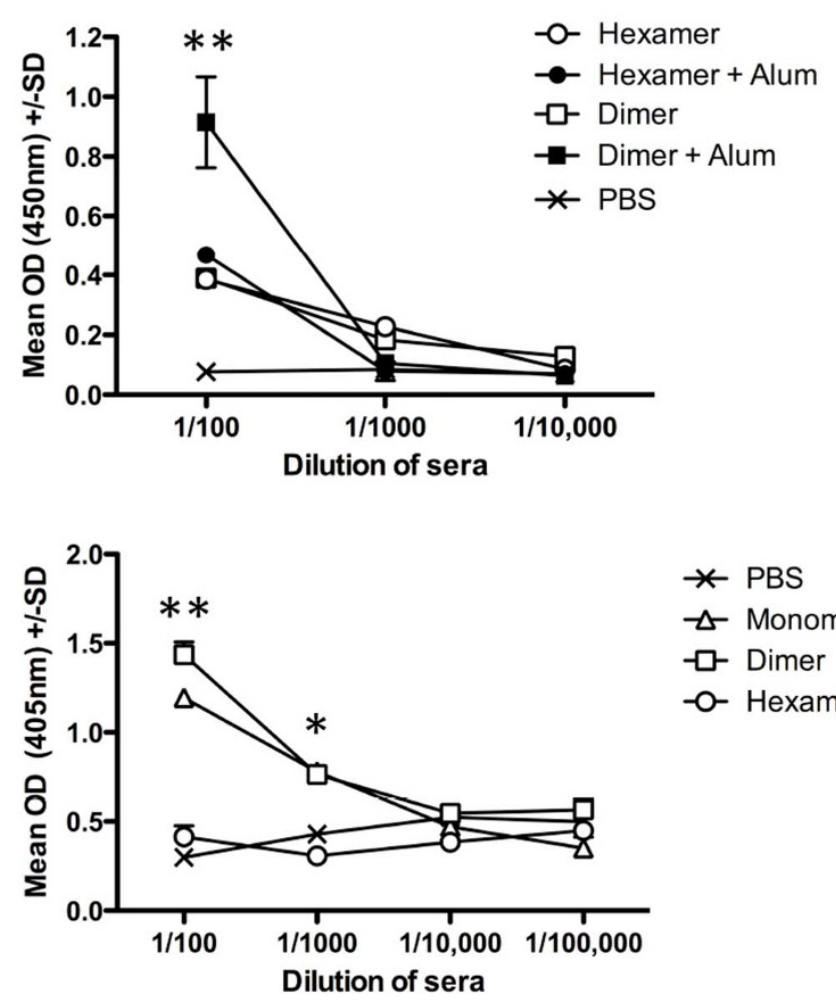

Figure 7 | Plasmodium falciparum MSP1 ${ }_{19}$-specific IgG1 Ab titres induced by immunization with hexameric or dimeric PfMSP1 19 -hIgG1-Fc-LH309/ 310CL-TP. (a) Groups of mice immunized with 3 doses of $10 \mu \mathrm{g} /$ animal at fortnightly intervals in the absence of adjuvant. Experiments were undertaken in $\mathrm{hFc} \gamma \mathrm{RI}$ transgenic BALB/c mice or their non-transgenic BALB/c control littermates (WT). (b) A repeat experiment using 4 animals per group and a total dose of $75 \mu \mathrm{g} / \mathrm{animal}$ of either hexamer or dimer in a 1:1 volume with Alum or PBS, in the hFc $\gamma \mathrm{RI}$ transgenic BALB/C mice. (c) Mouse anti-human IgG1 responses from mice immunized with monomer, dimer, hexamer or PBS. Each point represents the mean optical density $(+/-$ SD) of duplicate wells for each mouse within a given group. Mann Whitney test comparing dimer vs hexamer vaccinated animals; $* *=\mathrm{P}<0.0001, *=\mathrm{P}<0.05$.

suggesting that the presence of the Fc is tolerogenic. Although the CE-His induced notable levels of IgE, no IgE whatsoever was detectable in the CE-mIgG2a-Fc-fusion immunized animals, showing that the presence of the mIgG2a-Fc, even in the context of adjuvant, was preventing an Ag-specific IgE response. As observed for the PfMSP $1_{19}$-IgG2a-Fc-fusion, immunization of mice with CEmIgG2a-Fc drove a strong IgG1 response, and like the malaria model, immunization with CE-mIgG2a-Fc-fusions did not protect animals from a challenge infection with the cercariae parasites (See supplementary Table 1 online). This trend to generate mIgG1 responses was seen with all the Fc-fusions irrespective of Ag, since a fusion to enhanced green fluorescent protein also induced mIgG1 (See supplementary Fig. 12 online). Therefore the Fc-scaffold, and not the Ag tagged to it, is critical for driving the skewed IgG1 responses observed in animals inoculated with the various Fc-fusions.
We next examined the immunogenic properties of the purified hexamer and dimer in immunization and challenge experiments with malaria parasites. We observed significantly lower PfMSP1 $1_{19^{-}}$ specific (Figure 7a,b), and a complete absence of anti-hIgG1-Fc (Figure 7c) responses from the hexamer. Immunization of the animals with either the hexameric or dimeric PfMSP1 19 -hIgG1-FcLH309/310CL-TP drove mIgG1 responses as seen with all the Fcfusion monomers (See supplementary Table 1 online). Although the PfMSP1 ${ }_{19}$-hIgG1-Fc-LH309/310CL-TP did bind weakly to hFc $\gamma$ RI (Figure 3), experiments in hFc $\gamma \mathrm{RI}$ (involved in phagocytosis of ICs) transgenic mice were not significantly different to wildtype littermates, suggesting that the poor Ab-titres observed with $P f \mathrm{MSP}_{19^{-}}$ hIgG1-Fc-LH309/310CL-TP are not mediated primarily via hFc $\gamma$ RI (Figure 7a), but by a more general inability to interact with Fc $\gamma \mathrm{R}$, FcRn and C1q. No improvement in Ag-specific Ab-titres, or resolution of malaria, were seen when immunizations with hexamer were 
repeated in the presence of alum (Figure $7 \mathrm{~b}$ and Supplementary Table 1 online).

\section{Discussion}

We have developed a broadly effective strategy to polymerize monomeric Fc-fusion proteins into well-defined hexameric polymers so as to modify the functional consequences of their interactions with effector systems. These hexameric constructs were indeed found to bind to key Fc $\gamma$ Rs differently than their monomeric and dimeric counterparts, though the strength of this interaction with the receptors was found to be dependent on the fusion partner. Beyond identifying this critical property for future development, we also identify constructs that for certain clinical applications would likely have significant advantages over existing strategies.

This dependence of the Fc-fusion-Fc $\gamma \mathrm{R}$ interaction strength on the fusion partner was unexpected, but was also observed with some of the monomeric-Fc-fusion-Fc $\gamma \mathrm{R}$ interactions as well. With the monomeric Fc-fusions, the interaction with hFcRn, hFc $\gamma \mathrm{RI}$, and $\mathrm{hFc} \gamma \mathrm{RIIA}{ }^{\mathrm{R} 131}$ was not significantly different whether the $P y \mathrm{MSP}_{42}$ $\mathrm{Ag}$ or the $P \mathrm{MMSP}_{19} \mathrm{Ag}$ was attached, but the interaction with hFc $\gamma$ RIIA $^{\mathrm{H} 131}$ and hFc $\gamma$ RIIIA/B was markedly different between these two fusions. The similar interaction with the former set indicates that each of the Fc-fusions is properly folded (as further demonstrated in supplemental Fig. 1), and suggests that the observed differences are owing to unique properties of the particular Ags and to different sensitivities of the receptors for these properties. The binding site for $\mathrm{hFcRn}$ on IgG is localized within the $\mathrm{C} \gamma 2-\mathrm{C} \gamma 3$ junction, while the binding site on IgG for the Fc $\gamma$ Rs localizes to the amino-terminal region of the $\mathrm{C} \gamma 2$ domains, within the lower hinge region ${ }^{24,25}$. Thus, the differences in affinity with the different Ags are likely owing to subtle differences in the attached Ag structure in the immediate vicinity of the lower hinge region to which the different Fc $\gamma$ Rs exhibit different sensitivities. It might thus be possible to exploit this sensitivity with different Ags to produce Fc-fusion proteins which exhibit even greater specificity in receptor activation. The tremendous impact of the type of fused Ag on binding to Fc $\gamma$ Rs and FcRn, independent of the constant Fc scaffold, highlights the importance of considering such binding effects for each individual Fcfusion.

With the polymeric Fc-fusions, while the dimeric fraction with the Pf MSP ${ }_{19}$ Ag interacted significantly with all of the hFc $\gamma \mathrm{R}$ investigated here as well as $\mathrm{hFcRn}$, the corresponding hexameric fraction bound poorly to all of these receptors with the exeption of the high affinity hFc $\gamma$ RI which bound weakly (Figure 3 ). These hexamers however clearly bound protein $G$ (during purification) and were recognized by Ag-specific and Fc-specific Abs (See supplementary Fig. 2 online), indicating that, as with the monomers, these hexameric Fc-fusions were properly folded, a finding supported by the SEC and AFM analyses (Fig. 1). The lack of affinity for these receptors was thus likely owing to the fused $P f M S P 1_{19} \mathrm{Ag}$. Indeed, in the absence of this Ag, the hexameric hIgG1-LH309/310CL-TP interacted markedly with hFc $\gamma$ RI, hFc $\gamma$ RIIA ${ }^{\mathrm{R} 131}$, hFc $\gamma$ RIIB, mFc $\gamma$ RI, mFc $\gamma$ RIIB, mFc $\gamma$ RIII (Figure 3), as well as with $\mathrm{mFcRn}$ but not with hFcRn (Figure 5). These latter findings are broadly in line with observations made for IgG1 ICs, which also bound appreciably to the low-affinity Fc $\gamma$ Rs compared to monomeric $\operatorname{IgG1} 1^{26}$. As the binding site for $\mathrm{Clq}$ has also been suggested to include regions within the hinge, if not within the $\mathrm{Fab}^{27}$, the lack of binding to $\mathrm{Clq}$ is probably also owing to the fused $P f \mathrm{MSP}_{19} \mathrm{Ag}$. Although we are presently unsure of the reason for this significant difference owing to the presence of the $\mathrm{PfMSP}_{19} \mathrm{Ag}$ in the hexameric polymer, we note that this $\mathrm{Ag}$ is exceptionally negatively charged (See supplementary Fig. 13 online), and so the increased overall negative charge of the hexamer, compared with the monomer and dimer, might contribute to the lower receptor affinity. Although the in vivo half-life of the hexameric PfMSP1 ${ }_{19}$-hIgG1-LH309/310CL-Fc-TP is shortened by the presence of the fused Ag (See supplementary Fig. 6 online), rapid or intermediate clearance may also be favorable in some applications for such constructs, as demonstrated for IgG immunoconjugates approved for cancer imaging and therapy ${ }^{28}$.

Although not investigated in the present work, the increased avidity binding to inhibitory Fc $\gamma$ RIIB observed for hexameric hIgG1-FcLH309/310CL-TP and the enhanced binding of hexameric PfMSP1 19 -hIgG1-Fc-LH309/310CL-TP to B lymphocytes could be of immediate benefit in the development of well-defined replacements for IVIG therapy. Seventy percent of prescribed IVIG is now used for the treatment of autoimmune inflammatory conditions, including idiopathic thrombocytopenic purpura (ITP), instead of as replacement therapy in patients with immune deficiency ${ }^{29}$. IVIG is purified from the pooled plasma of $\sim 3,000$ anonymous donors and as a consequence of extensive screening for viruses is expensive to make. Although the mechanisms of action for IVIG remain unclear, the most compelling support a role for the Fc portion of $\mathrm{Ab}$ and interactions with both inhibitory and activating Fc $\gamma$ Rs found on monocytes and macrophages since Fc-fragments derived from IVIG can cure children suffering from ITP $^{30}$, although the exact receptors involved are hotly debated ${ }^{31-34}$ and may vary for the disease for which IVIG is used ${ }^{35}$. Such beneficial effects occur rapidly in ITP where they are unlikely to be wholly dependent on the long half-life influenced by interactions with the $\mathrm{FcRn}^{5-7}$. It has also recently been shown that it is the polymeric fraction, and more specifically the sialic acid component of IVIG that is responsible for this beneficial effect $^{31,36}$, observations supported by the reversal of ITP in mice by ICs or deglycosylated IVIG ${ }^{32,37}$. It is known that IVIG binds to CD22 lectin (Siglec-2) on B lymphocytes via a terminal sialic acid on the Fcglycan $^{38}$, and a recent study demonstrated that sialylated Fcs are responsible for the anti-inflamatory effects of IVIG in a mouse model of arthritis as a result of their interaction with human lectin receptor, DC-SIGN, on DCs ${ }^{39}$. Because the proportion of active multimeric IgG and/or sialylated IgG in commercially available IVIG is extremely low ( $<1 \%$ and $5 \%$ respectively), very large doses of IVIG have to be given to patients, typically 2 g per kg bodyweight, and this can cause adverse reactions in some patients. Although the exact nature and molecular make-up of the polymeric fraction of IgG in IVIG preparations remains unclear, we have confirmed that the polymeric Fc-fusions described here are sialylated (See supplementary Fig. 14 online). The enhanced binding of hexameric PfMSP1 19 -hIgG1-FcLH309/310CL-TP to B lymphocytes is particularly intriguing in this regard, since this binding is not effected by Fc $\gamma R$ or FcRn. These hexameric Fc-fusions may therefore cluster sialic acid binding receptors and impart protection from autoimmune disease more effectively, and at lower dosages, than currently seen with IVIG. Although many IVIG preparations contain dimers that arise from idiotype (Id)/anti-Id complexes associating non-covalently through Fab interactions ${ }^{40}$, they may also contain covalent IgG2 dimers ${ }^{41}$, suggesting that exploration of polymeric Fc-fusions on an IgG2 backbone may be usefully investigated given that hIgG2, as with hIgG1-FcLH309/310CL-TP, can interact with hFc $\gamma$ RIIA $^{\text {R131 }} \cdot{ }^{26,42}$ Although larger $(>350 \mathrm{kDa})$ multimeric IgG and circulating ICs are mostly removed in the preparation of IVIG, these are common in healthy individuals where they can contribute as much as $10 \%$ to the overall plasma Ab concentration (See supplementary Fig. 15 online), indicating a physiological role in maintaining immune homeostasis in these healthy individuals ${ }^{43}$. These findings suggest that further work now needs to be done with the hexameric hIgG1-Fc to determine if it has potential for treating autoimmune disease, including ITP.

Most Fc-fusions are currently based on hIgG1 backbones that can only engage with activating Fc $\gamma$ Rs expressed by innate immune cells including phagocytes ${ }^{5-7,25,26}$, and therefore this class of Ab may not be the ideal scaffold for delivery of Ags to adaptive immune cells involved in generating long term memory responses by useful vaccines. Indeed the Fc-fusions described here induced significant, yet 
non-protective, MSP1 $1_{19}$-specific $\mathrm{mIgG} 1$ responses in immunization and challenge studies with malaria parasites (See supplementary Table 1 online). Mouse IgG1 is believed not to be a subclass associated with protective properties as it possesses an extremely low activation to inhibition ratio ( 0.1 compared to 69 and 7 for IgG2a and IgG2b, respectively), as a consequence of its preference for binding inhibitory Fc $\gamma$ RIIB receptor ${ }^{44}$. Fusions of an unrelated Ag, cercarial elastase (CE) from S. mansoni, to the mIgG2a-Fc also generated non-protective IgG1 in wild-type mice (See supplementary Table $1 \&$ supplementary Fig. 10 online) and were likewise poorly immunogenic when compared with Ag administered in the absence of the Fc. We have shown here that the presence of the fused Ag can greatly interfere with the ability of the IgG1 polymers to engage Fc $\gamma$ Rs. Increasing the distance between the Ag and the $\mathrm{Fc} \gamma \mathrm{R}$-binding region on the $\mathrm{Fc}$, for example by making fusions to hIgG3 which has an extended hinge-region, may allow for polymers to interact with Fc $\gamma$ Rs without steric hindrance from the fused protein. Alternatively the use of smaller Ags or peptides incorporating B and $\mathrm{T}$ cell epitopes may prove more effective.

In conclusion, the prevention of infectious diseases, allergies, malignancies, fertility and immune disorders by vaccination requires immune-potentiators and suitable delivery systems that together constitute the adjuvant responsible for triggering optimal immune responses. Here we have modified the hugely successful IgG Fcfusion platform for the simultaneous delivery of multiple Ags and/ or ligands to the immune system that may mimic the 'depot-effect' of ICs, whilst removing the need for the vaccine formulation to be associated with toxic adjuvants. While not tested here, this modification is also expected to enhance the efficacy of existing Fc-based biologicals, which have already shown tremendous promise as monomeric constructs.

\section{Methods}

Construction of monomeric Fc-fusion vectors. Primer pairs containing EcoRI and BglII restriction sites: 5' -AATAATGAATTCCAACATTGCCCAACACCAATG-3' and 5' -AATAATAGATCTGTTAGAGGAAGAACAGAAGAT-3' were designed to amplify a codon-optimized PfMSP $1_{19}$ from plasmid PUCPfMSP $1_{19}$ into a TOPO TA parking vector by polymerase chain reaction (PCR) in order to create pTOPOTA2.1PfMSP1 $1_{19}$. The PfMSP1 $1_{19}$ was then sub-cloned as an EcoRI/BglII fragment into pFUSE vectors (Invivogen) containing the hIgG1 or mIgG2a Fc to create pFUSE-PfMSP $1_{19}$-hIgG1-Fc and pFUSE-PfMSP $1_{19}$-mIgG2a-Fc respectively. Cercarial elastase (CE) from Schistosoma mansoni was sub-cloned from pGEXEL $^{45}$ as a BglII fragment by PCR using primer pairs $5^{\prime}$-ATCTGGTTCCGCGTAGATCTATACGTAGTGGTGA-3' and 5' -AATTAAGCTGGAGATCTAATATTGGAGCGTACAA- $3^{\prime}$, and ligated into pFUSE-mIgG2a to create pFUSE-SmCEmIgG2a-Fc. Enhanced Green Fluorescent Protein (EGFP) was sub-cloned as a EcoRI/ BglII fragment from the plasmid pEGFP-C2 (BD Biosciences) by PCR using primer pairs 5' -TATAGAATTCCATGGTGAGCAAGGGCGAGGAGCT-3' and 5' TATAAGATCTCTTGTACAGCTCGTCCATGCCGA-3' and ligated into pFUSEmIgG2a-TP

Construction of polymeric Fc-fusion vectors. To generate a polymeric Ag Fc-fusion protein, three step changes to the coding sequence of the hIgG1 Fc-portion were made. The 18 amino-acid tailpiece from hIgM was sub-cloned onto the C-terminus of the $\mathrm{Fc}$ portion, and an additional mutation in the $\mathrm{C} \gamma 2$ domain to convert residues 309 and 310 (EU numbering throughout) to cysteine and leucine respectively. In order to insert the hIgM tailpiece sequence into the pFUSE vector, primer pairs 5' CTAGGACCCCCCCTGTACAACGTGTCCCTGGTCATGTCCGACACAGCTGGCACCTGCTACTGAG-3' and 5' -CTAGCTCAGTAGCAGGTGCCAGCTGTGTCGGACATGACCAGGGACACGTTGTACAGGGGGGGTC-3' were designed which would, when annealed together, form a double stranded sequence with overhanging bases encoding a NheI restriction site to allow sub-cloning C-terminal to the pFUSE vector. In order to maintain the reading frame of the protein encoded by the plasmid, remove an existing stop codon, and allowing for convenient restriction sites, an extra DNA base was inserted. The pFUSE vector was then digested with the restriction enzyme NheI, and the hIgM tailpiece insert above ligated to create an intermediary plasmid. In order to allow the hIgM tailpiece to be translated after the Fc region, the stop codon present was mutated in a subsequent step, via site directed mutagenesis, using the Quick Change II Kit (Stratagene, La Jolla, CA, USA) and primers pairs 5' - CTGTCTCCGGGTAAATTAGTCCTAGGACCCCCCCTG-3' and 5' - CAGGGGGGGTCCTAGGACTAATTTACCCGGAGACAG-3' to create an AvrII restriction enzyme site between the Fc region and the hIgM tailpiece. This created the plasmid termed pFUSE-hIgG1-Fc-TP. When transfected into mammalian cells this construct did not induce polymerization; therefore we made additional changes to mimic hIgM by mutating the cysteine at position 309, involved in forming a disulphide bridge between two monomers of IgM within the pentamer. In order to better mimic the protein sequence of hIgM, primer pairs $5^{\prime}$ GTGGTCAGCGTCCTCACCGTCTGCCTCCAGGACTGGCTGAATGGCAAG- $3^{\prime}$ and $5^{\prime}$ CTTGCCATTCAGCCAGTCCTGGAGGCAGACGGTGAGGACGCTGACCAC- ${ }^{\prime}{ }^{\prime}$ were designed to introduce a cysteine residue at position 309 , again by site-directed mutagenesis, as before. Upon alignment of the nucleotide sequence encoding the protein sequence of hIgM with that of human IgG1-Fc, it was decided, in addition, to replace the neighbouring histidine residue at position 310 with a neutral leucine residue. The final plasmid incorporating both mutations and the $P f \mathrm{MSP}_{1}{ }_{19} \mathrm{Ag}$ was named pFUSEPfMSP1 19-hIgG1-Fc-TP-LH309/310CL. To verify incorporation of the desired mutations and to check for PCR-induced errors, the entire coding sequences of all the expression vectors were sequenced.

A similar three-step strategy was used to generate pFUSE-PfMSP1 $1_{19}-\mathrm{mIgG} 2 \mathrm{a}-\mathrm{Fc}$ TP and pFUSE-PfMSP1 $1_{19}$-mIgG2a-Fc-TP-QH309/310CL with the following first, second and third step primer pairs respectively, primer pair $1: 5^{\prime}$ -

CTAGGCCCCCCCTGTACAACGTGTCCCTGGTCATGTCCGACACAGCTGGCACCTGCTACTGAG- ${ }^{\prime}$ and $5^{\prime}$-CTAGCTCAGTAGCAGGTGCCAGCTGTGTCGGACATGACCAGGGACACGTTGTACAGGGGGGGC-3'; primer pair 2: $5^{\prime}$ CGGTCTCTGGGTAAATTACCTAGGCCCCCCCTGTAC- $3^{\prime}$ and $5^{\prime}$ GTACAGGGGGGGCCTAGGTAATTTACCCAGAGACCG-3'; primer pair 3: $5^{\prime}$ GTCAGTGCCCTCACCGTCTGCCTCCAGG-3' and 5' CCTGGAGGCAGACGGTGAGGGCACTGAC-3'

Construction of IgM-Fc fusions. The hIgM-Fc was amplified as a BglII/NheI fragment by PCR using C $\mu 2$-For $5^{\prime}$

GTTAGATCTATTGCCGAGCTGCCTCCCAAA- ${ }^{\prime}$ and C $\mu 4-\operatorname{Rev} 5^{\prime}$ AGGCTAGCTGCATGCACACACAGAGC- ${ }^{\prime}$ ' primer pairs from plasmid pUC $\mu^{19,20}$. The PCR product was cloned in place of hIgG1-Fc in pFUSE-hIgG1-Fc-TP to generate pFUSE-IgM-Fc. PfMSP $1_{19}$ or $P y \mathrm{MSP} 1_{19}$ was released as an EcoRI/BglII fragment from $P f M S P 1_{19}$-hIgG1-Fc or $P y M S P 1_{19}$-hIgG1-Fc and cloned into the same restriction sites in pFUSE-IgM-Fc to form $P f M S P 1_{19}-\operatorname{IgM}-\mathrm{Fc}$ and $P y \mathrm{MSP}_{19^{-}}$ IgM-Fc respectively. $P y \mathrm{MSP}_{42}$ was generated by PCR using primer pairs $5^{\prime}$ CGGAATTCGTCCGAAGATGCACCAGAAA-3' and $5^{\prime}$-CGAATTCGATCCCATAAA GCTGGAAGAACT-3' from plasmid $P y \mathrm{MSP}_{42}$, and sub-cloned as an EcoRI fragment into pFUSE-IgM-Fc to generate $\mathrm{pPyMSP} 1_{42}$-IgM-Fc.

Expression, purification, and characterization of Fc-fusions. $\mathrm{CHO}-\mathrm{K} 1$ cells (European Collection of Cell Cultures) were transfected by electroporation with plasmids and positive clones selected. The cells were grown in DMEM complete media supplemented with $10 \%$ ultra-low bovine IgG FCS, $100 \mathrm{IU} / \mathrm{ml}$ penicillin, and $100 \mu \mathrm{gml}^{-1}$ streptomycin (PAA) at $37^{\circ} \mathrm{C} / 5 \% \mathrm{CO}_{2}$. Stable transfectants were selected in medium containing $400 \mathrm{\mu gml}^{-1}$ of Zeocin (Invivogen). Clones secreting Fc-fusion proteins were detected by sandwich enzyme-linked immunosorbent assay (ELISA) using mAbs 12.10 or 12.8 to capture $P f \mathrm{MSP}_{19}$ followed by goat anti-human IgG-Fc (Sigma-Aldrich: A0170) or goat anti-mouse IgG-Fc (Pierce: 31439 ) Abs conjugated to horseradish peroxidase (-HRP). From large-scale cultures in DMEM supplemented with ultralow IgG containing FBS (Gibco), Fc-fusion proteins were purified on Protein-G-Sepharose (GE Healthcare, Little Chalfont, Bucks, UK) or on anti-human IgM agarose (Sigma). Eluted fractions from the affinity purification were pooled and separated by size-exclusion chromatography on a high-performance Superdex-200

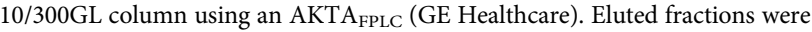
compared against known high MW gel filtration standards (Biorad). The integrity of the proteins was verified by SDS-PAGE on native $6 \%$ Tris-glycine gels for polymers or NuPAGE $4-12 \%$ bis-Tris acrylamide gels for monomers, against SeeBlue2 prestained molecular weight markers (Novex-Invitrogen).

Surface Plasmon Resonance (SPR) analysis. SPR experiments were carried out using a Biacore 3000 instrument (GE Healthcare). Flow cells of CM5 sensor chips were coupled with recombinant forms of soluble human or mouse FcRn (hFcRn or mFcRn; 2200-3000 RU) using amine coupling chemistry as described in the protocol provided by the manufacturer. The coupling was performed by injecting $2 \mu \mathrm{gml}^{-1}$ of the protein in $10 \mathrm{mM}$ sodium acetate $\mathrm{pH} 5.0$ (GE healthcare). Phosphate buffer (67 $\mathrm{mM}$ phosphate buffer, $0.15 \mathrm{M} \mathrm{NaCl}, 0.005 \%$ Tween 20 ) at $\mathrm{pH} 6.0$, or HBS-EP buffer (0.01 M HEPES, $0.15 \mathrm{M} \mathrm{NaCl}, 3$ mM EDTA, 0.005\% surfactant P20) was used as running buffer and dilution buffer. $100 \mathrm{nM}$ of each of the Fc-fusion proteins were injected over immobilized receptor at $\mathrm{pH} 6.0$ or $\mathrm{pH} 7.4$, with a flow rate of $20 \mu \mathrm{l} / \mathrm{min}$ at $25{ }^{\circ} \mathrm{C}$. Regeneration of the surfaces was done using injections of HBS-EP buffer at pH 7.4 (GE Healthcare). In all experiments, data were zero adjusted and the reference cell subtracted. Data were evaluated using the BIAevaluation 4.1 software (GE Healthcare).

Enzyme linked immunosorbent assays (ELISA). Microtiter wells (Nunc) were coated with titrated amounts of the Fc-fusions (50-0.3 nM) in PBS or carbonate buffer pH9 and incubated over night at $4{ }^{\circ} \mathrm{C}$ prior to blocking with $4 \%$ skimmed milk (Acumedia) for $1 \mathrm{~h}$ at room temperature (RT). The wells were washed four times with PBS/0.005\% Tween 20 (PBS/T) pH 7.4 before addition of GST or HIS-fused hFc $\gamma$ RI, hFc $\gamma$ RIIA, hFc $\gamma$ RIIB, mFc $\gamma$ RI, mFc $\gamma$ RIIB diluted in $4 \%$ skimmed milk PBS $/ 0.005 \%$ Tween 20 (PBS/T) pH 7.4 and added to the wells. After incubation for $2 \mathrm{~h}$ at RT and washing as above, an HRP-conjugated polyclonal anti-GST from goat (1:8000; GE Healthcare) was added and incubated for $1 \mathrm{~h}$ at RT. Wells were washed as above, $100 \mu \mathrm{l}$ of the substrate TMB (Calbiochem) was added to each well and incubated for 
45 minutes before $100 \mu \mathrm{l}$ of $0.25 \mathrm{M} \mathrm{HCl}$ was added. The absorbance was measured at $450 \mathrm{~nm}$ using a Sunrise TECAN spectrophotometer (TECAN, Maennedorf, Switzerland).

Flow cytometry. Human leucocytes were purified from whole blood by centrifugation on Polymorphoprep gradients according to manufacturers instructions. $1 \times 10^{5}$ cells were incubated with $200 \mu \mathrm{l}$ FACs buffer (phosphatebuffered saline, $0.2 \%$ bovine serum albumin, $5 \%$ goat serum) containing $40 \mu \mathrm{g}$ of Fcfusions or buffer only as indicated for $1 \mathrm{~h}$ at room temperature. Cells were washed twice with FACs buffer and incubated for $1 \mathrm{~h}$ at $4{ }^{\circ} \mathrm{C}$ with $1 / 500$ dilution of $\mathrm{F}\left(\mathrm{ab}^{\prime}\right)_{2}$ goat anti-hIgG-Fc-phycoerythrin (PE) or goat anti-hCD19-fluorescein isothiocyanate (FITC)-conjugated Abs (Southern Biotechnology) in $200 \mu$ l FACs buffer. After washing with FACs buffer, cells were analyzed on a FACScan (BD Biosciences). Data acquisition was conducted with CELLQuest software (BD Biosciences) and the analysis performed with FlowJo version 9.1.

Ag-specific IgG subclass and Complement binding analysis. ELISA plates were

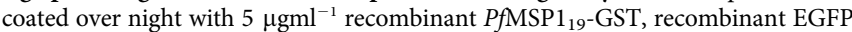
(Biovision), native CE from cercarial transformation fluid, or $20 \mu \mathrm{g} \mathrm{ml}^{-1} \mathrm{hIgG1}$ diluted in carbonate buffer, $\mathrm{pH}$ 9.0. The next day ELISA plates were blocked in PBS$0.5 \%$ Tween-20 containing 5\% milk powder (Marvel). After three washes $100 \mu \mathrm{l}$ of varying dilutions of sera in DMEM/10\% FBS were added to duplicate or triplicate wells and $\mathrm{Ab}$ allowed to bind for $1 \mathrm{~h}$. After 3 washes in PBS-Tween-20,100 $\mu \mathrm{l}$ of $1: 1,000$ dilution of goat anti-mIgG1-HRP, IgG2a-HRP, IgG2b-HRP, IgG3-HRP, IgE-HRP or IgM-HRP (all Southern Biotech) in DMEM/10\% FBS were added and incubated for a further $1 \mathrm{~h}$. After 3 washes as above, plates were developed with $3,3^{\prime}, 5,5^{\prime}$-tetramethylbenzidine dihydrochloride (Sigma: T3405) in phosphate citrate buffer containing sodium perborate (Sigma: P4922). After $10 \mathrm{~min}$, colour development was stopped with $50 \mu \mathrm{l}$ of $2 \mathrm{M} \mathrm{H}_{2} \mathrm{SO}_{4}$ and the absorbance at $450 \mathrm{~nm}$ read using an ELISA plate reader (Ultramark, Microplate Imaging System, Biorad). C1q and C5-9 binding assays were essentially as described previously ${ }^{46}$.

Atomic force microscopy. Stock solutions of $P f M S P 1_{19}$-hIgM-Fc or hIgG1-FcLH309/310CL-TP in 1xHBSS buffer were diluted to $10 \mu \mathrm{gml}^{-1}$ in $0.2 \times H B S S$ buffer and then directly applied to a freshly cleaved fragment of muscovite mica. After incubating for 20 minutes, the sample was rinsed extensively with $0.2 x H B S S$ buffer to remove unadsorbed molecules. The samples were always under solution during transport to and imaging within the AFM. Imagaing was performed in the tapping mode with a Nanoscope IIIa Multimode AFM (Veeco, Santa Barbara, CA) using silicon nitride cantilevers (NP-S, Veeco Probes, Santa Barbara, CA) with a spring constant of $0.32 \mathrm{~N} / \mathrm{m}$ in $0.5 \mathrm{xHBSS}$ buffer. The typical scan rate and initial oscillation amplitude were $3 \mathrm{~Hz}$ and $20 \mathrm{~nm}$, respectively, and the applied force was minimized to $\sim 0.1 \mathrm{nN}$. The piezoscanner ( $9 \mu \mathrm{m}, \mathrm{D}$ scanner, Veeco) was calibrated using mica and gold ruling. All images here were reproducible with different tips and different fastscan directions. All lateral dimensions were determined from the full-width at half height.

Molecular modelling. The monomer was constructed from the crystal structure of $P f \mathrm{MSP}_{19}$ and the hIgG1 Fc-domain ${ }^{47,48}$, using a random chain structure for both the hinge and tailpiece regions. Each monomer was energy minimized before being assembled into the hexameric complex. All energy calculations were performed using VMD/NAMD ${ }^{49,50}$ using the CHARMM27 force field ${ }^{51}$. NAMD was developed by the Theoretical Biophysics Group in the Beckman Institute for Advanced Science and Technology at the University of Illinois at Urbana-Champaign. To construct the hexamer, we took advantage of two experimental observations: first, the stoichiometry of the complex is finite (that is, oligomerization does not proceed indefinitely), and second, oligomerization required the Cys309 (here, C207) mutation. The former observation suggests that there is some physical reason preventing further assembly, which we reasoned was owing to the complex being ring-shaped, similar to those seen with natural IgM complexes ${ }^{21}$. The latter observation suggests that adjacent monomers are linked through these $\mathrm{C} 309$ residues. For these linkages to be possible in a ring-shaped complex, these residues must all lie within a common plane, as is the case with the homologous cysteine residues in the IgM pentamer ${ }^{21}$. With these Fc-fusions, there are two types of oligomers that can satisfy these criteria: star-shaped, in which the $\mathrm{C} 309$ plane is the same as the Fc-plane, and barrel-shaped, where the C309 plane is perpendicular to the Fc-plane. Both models were examined to determine which produced a structure with lower energy, subject to one additional criterion. Since these Fc-fusions have not evolved to interact with each other but rather link by virtue of the mutated C309 residues, we assumed that the monomers do not undergo significant structural changes to form these bonds, and so assigned an energetic penalty to any such changes. This was achieved by constraining the backbone atoms within a harmonic well (spring constant, $1 \mathrm{kcal} /$ $\mathrm{mol} / \AA^{2}$ ) centered on the positions of the energy-minimized monomeric structures. By monitoring the contribution of this constraining potential to the total minimized energy, the extent to which each monomer changed conformation from the monomeric form could be evaluated. Without this constraint, the star-shaped and barrel-shaped structures, evaluated at a range of initial C309-C309 distances, were of a similar minimized energy. However with the constraint, at all distances, it was clear that the barrel-shaped structure required significantly fewer structural changes than the star-shaped model to form the C309-C309 disulfide bridge. Thus, without the constraint, the barrel-shaped structure achieves a similar minimized energy as the star-shaped structure, but requiring fewer changes to the monomeric form. We therefore favor a barrel-shaped structure for the hexamer. The final model was constructed at the closest initial C309-C309 distance at which there was the smallest degree of structural changes to the monomeric structure. These calculations were performed without any of the tailpieces connected to other monomers. With this minimized model, the tailpieces were connected to other, randomly selected, monomers within the complex, and then the entire complex was solvated with TIP water ${ }^{52}$, minimized, and finally equilibrated, as judged by the root-mean-squareddeviation of the protein backbone. The electrostatic potential was calculated using Swiss-PdbViewer ${ }^{53}$. The results shown in supplementary Fig. 13 (online) were calculated using the Coulomb approximation but similar results were obtained calculating the Poisson-Boltzmann equation.

Mice, parasites and immunization schedule. Sex and age-matched groups of 4-6 $\mathrm{BALB} / \mathrm{C}$ mice from 8 weeks of age, bred under specific pathogen-free conditions, were immunized with Fc-fusions at $10 \mu \mathrm{g} /$ dose (unless stated otherwise) administered by sub-cutaneous (s.c.) or intraperitoneal (i.p.) injection at two week intervals on three separate occasions, either alone or suspended with Alum (Thermo Scientific: 77161) as a clinically relevant adjuvant, and as a baseline for efficacy of conventional immunization strategies alone. Blood was collected by tail bleeding 2 weeks after the last immunization and ahead of challenge with parasites in order to assess levels of Ag-specific Ab prior to infection. Mice were challenged with 10,000 infected erythrocytes of Plasmodium berghei transgenic for PfMSP $1_{19}$ as described previously ${ }^{54}$, or with 200 cercariae from a Puerto Rican isolate of Schistosoma manson $i^{45}$. To determine the in vivo half-life, groups of 4 female Balb/C mice were injected i.p. with $300 \mu \mathrm{g}$ of PfMSP119-hIgG1-Fc-LH309/310CL-TP or GammaGard $^{\mathrm{TM}}$ (Baxter Healthcare). Mice were bled at routine intervals and levels of Fc-fusion determined by ELISA on pooled sera at a $1: 10$ dilution against known standards by ELISA as described above.

1. Strohl, W. R, Knight, D. M. Discovery and development of biopharmaceuticals: current issues. Curr Opin Biotech 20, 668-672 (2009).

2. Huang, C. Receptor-Fc fusion therapeutics, traps, and MIMETIBODY ${ }^{\mathrm{TM}}$ technology. Curr Opin Biotech 20, 692-699 (2009).

3. Roopenian, D. C. \& Akilesh, S. FcRn: the neonatal Fc receptor comes of age. Nat Rev Immunol 7, 715-25 (2007).

4. Vaccaro, C., Bawdon, R., Wanjie, S., Ober, R. J. \& Ward, E. S. Divergent activities of an engineered $\mathrm{Ab}$ in murine and human systems have implications for therapeutic Abs. Proc Natl Acad Sci USA 103, 18709-14 (2006).

5. Nimmerjahn, F. \& Ravetch, J. V. Fc-receptors as regulators of immune responses. Nat Rev Immunol 8, 34-47 (2008)

6. Nimmerjahn, F. \& Ravetch, J. V. Fc-receptors as regulators of immunity. Adv Immunol 96, 179-204 (2007).

7. Nimmerjahn, F. \& Ravetch, J. V. Fc $\gamma$ Rs in health and disease. Curr Top Microbiol Immunol 350, 105-25 (2011).

8. Regnault, A. et al. Fc $\gamma$-receptor-mediated induction of dendritic cell maturation and $\mathrm{MHC}$ class I restricted $\mathrm{Ag}$ presentation after immune complex internalization. J Exp Med 189, 371-80 (1999).

9. Dhodapkar, K. M., Krasovsky, J., Williamson, B., Dhodapkar, M. V. Antitumour monoclonal Abs enhance cross-presentation of cellular Ags and the generation of myeloma-specific killer T cells by dendritic cells. J Exp Med 195, 125-33 (2002).

10. Groh, V. et al. Efficient cross-priming of tumour Ag-specific T cells by dendritic cells sensitized with diverse anti-MICA opsonized tumour cells. Proc Natl Acad Sci USA 102, 6461-66 (2005)

11. Rafiq, K., Bergtold, A. \& Clynes, R. Immune complex-mediated Ag presentation induces tumor immunity. J Clin Invest 110, 71-9 (2002).

12. Schuurhuis, D.H, et al. Immune complex-loaded dendritic cells are superior to soluble immune complexes as antitumor vaccine. J Immunol 176, 4573-80 (2006)

13. Baker, K. et al. Neonatal $F \mathrm{c}$ receptor for IgG $\left(F_{c} R n\right)$ regulates cross-presentation of IgG immune-complexes by CD8-CD11b+ dendritic cells. Proc Natl Acad Sci USA 108, 9927-32 (2011).

14. Bergtold, A., Desai, D. D., Gavhane, A. \& Clynes, R. Cell surface recycling of internalized Ag permits dendritic cell priming of B cells. Immunity 23, 503-14 (2005).

15. Kalergis, A. M. \& Ravetch, J. V. Inducing tumor immunity through the selective engagement of activating Fc $\gamma$-receptors on dendritic cells. J Exp Med 195, 165359 (2002)

16. Boruchov, A. M. et al. Activating and inhibitory IgG Fc receptors on human DCs mediate opposing functions. J Clin Invest 115, 2914-23 (2005).

17. Dhodapkar, K. M. et al. Selective blockade of inhibitory Fcg-receptor enables human dendritic cell maturation with IL-12p70 production and immunity to Abcoated tumor cells. Proc Natl Acad Sci USA 102, 2910-15 (2005).

18. Smith, R. I., Coloma, M. J. \& Morrison, S. L. Addition of a mu-tailpiece to IgG results in polymeric Abs with enhanced effector functions including complementmediated cytolysis by IgG4. J Immunol 154, 2226-36 (1995).

19. Sœrensen, V., Sundvold, T. E., Michaelsen, T. E. \& Sandlie, I. Polymerization of IgA and IgM: roles of Cys309/Cys414 and the secretory tailpiece. J Immunol 162, 3448-55 (1999).

20. Ghumra, A. et al. Identification of residues in the $\mathrm{C} \mu 4$ domain of polymeric IgM essential for interaction with Plasmodium falciparum erythrocyte membrane protein 1 (PfEMP1). J Immunol 181,1988-2000 (2008). 
21. Czajkowsky, D. M. \& Shao, Z. The human IgM pentamer is a mushroom-shaped molecule with a flexural bias. Proc Natl Acad Sci USA 106, 14960-65 (2009).

22. Andersen, J. T., Daba, M. B., Berntzen, G., Michaelsen, T. E. \& Sandlie, I. Crossspecies binding analyses of mouse and human neonatal $F c$ receptor show dramatic differences in IgG and albumin binding. J Biol Chem 285, 4826-36 (2010).

23. Doenhoff, M. J. A vaccine for schistosomiasis: alternative approaches. Trends Parasitol 14, 105-9 (1998).

24. Woof, J. M., Partridge, L. J., Jefferis, R. \& Burton, D. R. Localization of the monocyte-binding region on human immunoglobulin G. Mol Immunol 23, 31930 (1986).

25. Woof, J. M. \& Burton, D. R. Human Ab-Fc receptor interactions illuminated by crystal structures. Nat Rev Immunol 4, 89-99 (2004).

26. Bruhns, P. et al. Specificity and affinity of human Fcgamma receptors and their polymorphic variants for human IgG subclasses. Blood 113, 3716-25 (2009).

27. Gaboriaud, C. et al. The crystal structure of the globular head of complement protein $\mathrm{Clq}$ provides a basis for its versatile recognition properties. J Biol Chem 278, 46974-82 (2003).

28. Wu, A. M. \& Senter, P. D. Arming Abs: prospects and challenges for immunoconjugates. Nat Biotech 23, 1137-46 (2005).

29. Orange, J. S. et al. Use of intravenous immunoglobulin in human disease: a review of eviodence by members of the Primary Immunodeficiency Committee of the American Academy of Allergy, Asthma and Immunology. J Allergy Clin Immunol 117, S525-53.

30. Debré $\mathrm{M}$ et al. Infusion of Fc gamma fragments for treatment of children with acute immune thrombocytopenic purpura. Lancet 342, 945-9 (1993).

31. Samuelsson, A., Towers, T. L. \& Ravetch, J. V. Anti-inflammatory activity of IVIG mediated through the inhibitory Fc receptor. Science 291, 484-6 (2001).

32. Bazin, R. et al. Reversal of immune thrombocytopenia in mice by cross-linking human immunoglobulin $\mathrm{G}$ with a high-affinity monoclonal Ab. Br J Haematol 135, 97-100 (2006).

33. Crow, A. R. et al. IVIg-mediated amelioration of murine ITP via FcgammaRIIB is independent of SHIP1, SHP-1, and Btk activity. Blood 102, 558-60 (2003).

34. Leontyev, D. et al. The inhibitory Fc $\gamma$ receptor is unecessary for IVIG efficacy. Nature Preceedings.http://precedings.nature.com/documents/4635/version/1 (2010)

35. Araujo, L. M. et al. Intravenous Ig inhibits invariant NKT cell-mediated allergic airway inflammation through FcgRIIIA-dependent mechanisms. J Immunol 186, 3289-93 (2011).

36. Machino, Y. et al. Effect of IgG interchain disulfide bond cleavage on efficacy of IVIg for immune thrombocytopenic purpura (ITP). Clin Exp Immunol. 162, 41524 (2010).

37. Anthony, R. M. et al. Identification of a receptor required for the antiinflammatory activity of IVIG. Proc Natl Acad Sci USA 105, 19571-78 (2008).

38. Séité, J. F. et al. IVIg modulates BCR signaling through CD22 and promotes apoptosis in mature human B lymphocytes. Blood 116, 1698-704 (2010).

39. Anthony, R. M., Kobayashi, T., Wermeling, F. \& Ravetch, J. V. Intravenous gammaglobulin suppresses inflammation through a novel $\mathrm{T}_{\mathrm{H}} 2$ pathway. Nature 475, 110-116 (2011).

40. Roux, K. H. \& Tankersley, D. L. A view of the human idiotypic repertoire. Electron microscopic and immunologic analyses of spontaneous idiotype-anti-idiotype dimmers in pooled human IgG. J Immunol 144, 1387-95 (1990).

41. Yoo, E. M., Wims, L. A., Chan, L. A. \& Morrison, S. L. Human IgG2 can form covalent dimmers. J Immunol 170, 3134-8 (2003).

42. Schneider-Merck, T. et al. Human IgG2 Abs against epidermal growth factor receptor effectively trigger $\mathrm{Ab}$-dependent cellular cytotoxicity but, in contrast to IgG1, only by cells of the myeloid lineage. J Immunol 512-20 (2010).

43. Nezlin, R. Circulating non-immune IgG complexes in health and disease. Immunol Lett 122,141-4 (2009).

44. Nimmerjahn, F. \& Ravetch, J. V. Divergent immunoglobulin G subclass activity through selective Fc receptor binding. Science 310, 1510-2 (2005).

45. Price, H. P., Doenhoff, M. J. \& Sayers, J. R. Cloning, heterologous expression and Agicity of a schistosome cercarial protease. Parasitology 114, 447-53 (1997).

46. Lewis, M. J., Wagner, B. \& Woof, J. M. The different effector function capabilities of the seven equine IgG subclasses have implications for vaccine strategies. $\mathrm{Mol}$ Immunol 45, 818-27 (2008).
47. Pizarro, J. C. et al. Crystal structure of a Fab complex formed with PfMSP1-19, the C-terminal fragment of merozoite surface protein 1 from Plasmodium falciparum: a malaria vaccine candidate. J Mol Biol 328, 1091-103 (2003).

48. Deisenhofer, J. Crystallographic refinement and atomic models of a human FC fragment and its complex with fragment B of protein A from Staphylococcus aureus at 2.9- and 2.8-A resolution. Biochemistry 20, 2361-70 (1981).

49. Humphrey, W., Dalke, A., Schulten, K. VMD: visual molecular dynamics. J Mol Graph 14, 27-38 (1986).

50. Phillips, J. C. et al. Scalable molecular dynamics with NAMD. J Comput Chem 26, 1781-802 (2005)

51. MacKerell, A. D., et al. All atom empirical potential for molecular modeling and dynamics studies of proteins. J Phys Chem B 102, 3586-616 (1998)

52. Jorgensen, W. L., Chandrasekhar, J., Madura, J. D., Impey, R. W. \& Klein, M. L. Comparison of simple potential functions for simulating liquid water. J Chem Phys 79, 926-935 (1983).

53. Guex, N. \& Peitsch, M. C. SWISS-MODEL and the Swiss-PdbViewer: an environment for comparative modeling. Electrophoresis 18, 2714-23 (1997).

54. McIntosh, R. S. et al. The importance of human FcgammaRI in mediating protection to malaria. PLoS Pathogens 18;3(5),e72 (2007).

55. Martin, W. L., West, A. P., Gan, L. \& Bjorkman, P. J. Crystal structure at 2.8 A of an FcRn/heterodimeric Fc complex: mechanism of $\mathrm{pH}$-dependent binding. Mol Cell 7, 867-77 (2001).

56. Sauer-Eriksson, A. E., Kleywegt, G. J., Uhlén, M., \& Jones, T. A. Crystal structure of the $\mathrm{C} 2$ fragment of streptococcal protein $\mathrm{G}$ in complex with the Fc domain of human IgG. Structure 3, 265-78 (1995).

57. Sondermann, P., Huber, R., Oosthuizen, V., \& Jacob, U. The 3.2-A crystal structure of the human IgG1 Fc fragment-Fc gammaRIII complex. Nature 406, 267-273 (2000)

\section{Acknowledgements}

This work was supported by a Royal Society Theo Murphy Blue Skies Award to R.P. J.H. and Z.S are supported by the National Basic Research Program of China (No.2007CB936000) and the Science and Technology Commission of Shanghai Municipality (1052nm07700, 10PI1405100). D.M.C. was supported by the Chinese Academy of Sciences Fellowships for Young International Scientists (Grant 2009YA1-1). D.N.A.M. was supported by a MRC studentship. M.E-F. was supported by the Egyptian government. J.T.A was supported by the Norwegian Research Council (Grant no. 179573/V40) and South-Eastern Norway Regional Health Authority (Grant no. 39375). Z.S. is supported by K.C. Wong Foundation (H.K.). We thank Dr A. Holder (MRC, Mill Hill) and Dr S. Draper (Jenner Institute, University of Oxford) for providing purified $P f \mathrm{MSP} 1_{19}$ for ELISAs and $P f \mathrm{MSP} 1_{19}$ and $P y \mathrm{MSP}_{42}$ containing plasmids. We acknowledge Baxter Healthcare for provision of IVIG (Gammagard). We thank Dr Karen Bunting for the use of size-exclusion columns and general advice on protein purification. We thank Helen Price, Katherine Francklow and Jon Sayers for provision of plasmids containing CE. The authors acknowledge the Liverpool NIHR Biomedical Research Centre high throughput screening facility for infrastructural support in this project.

\section{Author contributions}

R.P. conceived and designed the overall study. R.P., D.N.A.M., J.T.A., I.S., \& R.M. designed experiments and D.N.A.M., J.S., M.E.F., A.T., D.M.C., J.H., J.T.A. \& R.S. performed experiments. M.D. provided constructs for cloning S. mansoni cercarial elastase and maintained the parasite life cycle for immunization experiments. D.M.C., J.H., and Z.S performed the modeling. R.P., D.N.A.M. and D.M.C. wrote the paper. D.N.A.M. and D.M.C contributed equally to the manuscript.

\section{Additional information}

Supplementary information accompanies this paper at http://www.nature.com/ scientificreports

Competing financial interests: the authors declare no competing financial interests.

License: This work is licensed under a Creative Commons

Attribution-NonCommercial-ShareAlike 3.0 Unported License. To view a copy of this license, visit http://creativecommons.org/licenses/by-nc-sa/3.0/

How to cite this article: Mekhaiel, D.N.A. et al. Polymeric human Fc-fusion proteins with modified effector functions. Sci. Rep. 1, 124; DOI:10.1038/srep00124 (2011). 